\title{
Physics Problem-Solvers on Why They Generate Unprompted Diagrams
}

\author{
Michael Vignal ${ }^{1}$ and Bethany R. Wilcox ${ }^{1}$ \\ ${ }^{1}$ Department of Physics, University of Colorado, 390 UCB, Boulder, CO 80309
}

Previous work has shown that many physics students will draw unprompted diagrams while solving physics problems. These diagrams are distinct from prompted student-generated diagrams in that they tend to be smaller and contain fewer details, an unsurprising finding given that these two types of diagrams are generated for different reasons. In this study, we expand and elaborate on reasons physics problem-solvers generate diagrams and the differences between unprompted and prompted diagrams by analyzing a set of 10 interviews with undergraduates, graduates, postdocs, and faculty in physics. Interviewees were given several physics problems and diagramming tasks to anchor a subsequent conversation about diagramming while problem solving. In these conversations, participants reported that their unprompted diagrams help them: understand the situation and question; store, organize, and prioritize information; support solution strategies; and communicate information. Participants also shared their beliefs about the limitations of diagrams, including when they are not useful or stop being useful, as well as difficulties with generating diagrams. 


\section{INTRODUCTION AND BACKGROUND}

Generating sketches, graphs, and other diagrams is an integral part of physics problem solving and may be done to orient to the problem $[1,2]$, to aid in problem solving $[3,4]$, or as a means of communicating understanding $[5,6]$. For these reasons, diagramming is often explicitly taught in physics courses [7-11].

While many STEM education researchers have investigated how students interpret and use common or professional representations $[4,7,12-17]$, only a few have looked at student-generated diagrams $[3,4,6,18]$, especially pertaining to student problem-solving. Some researchers have found that, while unprompted, accurate force diagrams may help students solve force problems $[3,6]$, prompting for diagrams may interfere with student problem solving [6].

In a previous study $[18,19]$, we asked 19 undergraduate and graduate physics majors to solve 18 multiple-choice (MC) physics problems to investigate when and why students draw unprompted diagrams. We found that, when students drew diagrams, the diagram almost always preceded any algebra or other written work, suggesting students generally at least start generating diagrams to help orient to the problem. Depending on the problem, students sometimes also added information to their diagrams as they worked towards an answer, though we were surprised to find that students who revisited their diagrams were less likely to select a correct answer (28\%) than students who drew but did not revisit their diagrams ( $48 \%$ correct), though this may indicate students engage more with diagrams on difficult problems. Finally, while many of the MC problems could be solved geometrically, we observed only a handful of cases in which a student obtained an answer directly from a diagram. Interviewees also completed 6 explicit diagramming tasks. analyzing the impact of prompting on student diagrams, we found: students were about half as likely to draw axes on unprompted diagrams, which they primarily did when the prompt included coordinate information; the prompted diagrams were generally larger; and students were twice as likely to label units on their prompted diagrams.

From the previous study, we identified frequencies relating to orienting, solving, and communicating, but we did not gain much understanding about how the diagrams helped students do these things. As described in the next section, the current study used a modified subset of the previous study's MC questions and diagramming tasks (Fig. 1) to anchor conversations about these different reasons for diagramming: these conversations allow us to expand and nuance our previous findings.

\section{METHODS}

Our interview problems (Fig. 1) were modified from a previous set of interviews $[18,19]$. Many of these problems are not trivial to solve, even for expert physicists. We created 3 versions of the interview with different orderings of the MC problems (Table I) where the first

\section{Blocks}

A $10 \mathrm{~kg}$ block sits on top of a $20 \mathrm{~kg}$ block, which sits on the floor. Between the two boxes, the coefficient of both static and kinetic friction is 0.6 , and it is 0.25 between the floor and the bottom $(20 \mathrm{~kg})$ box. A $100 \mathrm{~N}$ force is applied horizontally to the top $(10 \mathrm{~kg})$ box. What is the magnitude of the net force on the bottom box?

\section{Lens}

A converging lens with a focal length of $1 \mathrm{~m}$ is placed $2 \mathrm{~m}$ from an object. What is the magnification $M$ of the image of the object?

\section{Collision}

A $50 \mathrm{~g}$ ball travelling at $8 \mathrm{~m} / \mathrm{s}$ hits a $500 \mathrm{~g}$ ball at rest, after which the $50 \mathrm{~g}$ ball is traveling perpendicular to its original trajectory at a speed of $6 \mathrm{~m} / \mathrm{s}$. What is the final speed of the $500 \mathrm{~g}$ ball?

\section{E-Potential}

What is the electric potential at a point $1 \mathrm{~m}$ from a $+2 \mathrm{C}$ charge, $2 \mathrm{~m}$ from a $-3 \mathrm{C}$ charge, and $3 \mathrm{~m}$ from a $+1 \mathrm{C}$ charge? Mirrors

Two flat square mirrors are placed edge to edge with a $55^{\circ}$ angle between their surfaces. Light comes in, bounces off of the surface of each mirror exactly once, and then leaves the system of mirrors. What is the angle between the incoming and outgoing rays of light?

\section{E-Field}

A charge of $-q$ sits at $(\ell, 0,0)$ and a second charge $2 q$ sits at $(0, \ell, 0)$. What is the electric field at $(0,0, \ell)$ ? Mirrors Diagramming Task

Carefully draw and label a ray-diagram for a ray of light that bounces off two mirrors with an angle of $135^{\circ}$ between them. Collisions Diagramming Task

Carefully draw and label a collision where a disk of mass $m$, sliding on a frictionless surface, is deflected 60 degrees from its original trajectory after striking a larger mass $M$ that was at rest.

\section{E-Field Diagramming Task}

Carefully draw and label 3 points: $A$ at $(\ell, 0,0), B$ at $(0,-\ell, 0)$ and $C$ at $(0,0, \ell)$. Then, if a charge $-q$ sits at $A$ and a charge $3 q$ sits at $B$, sketch the electric field at the point $C$.

FIG. 1. Prompts for the 6 multiple-choice (MC) problems (answer-options not shown) and 3 explicit diagramming tasks.

two problems had no diagramming prompt, the next two came with a diagram, and the final two included the explicit diagramming prompt "Please include a detailed diagram with your answer." These 3 versions were created to help us investigate how different diagramming prompts impacted interviewees' diagramming and problem solving, an analysis that will require additional data and is beyond the scope of this article. Unlike in the previous study, interviewees were instructed to speak aloud as they worked through the problems [20]. Both sets of interviews covered a variety of physics content areas to minimize the impact of any peculiarities of a specific content area or type of problem on our data. After completing the prompts, or if time was running short, the interviewer switched to the follow-up, semi-structured [21] discussion about why the interviewee (in general) generates diagrams and the role(s) of these diagrams in physics problem-solving. This discussion also involved reviewing the diagrams the interviewees had drawn.

Interviews took place during the 2020-2021 academic year and were conducted over Zoom, with the interviewee 
TABLE I. Breakdown of the 3 different interview versions, including pseudonyms for interviewees who were given each version. All three interview versions had the same 3 explicit diagramming tasks (in the same order) as shown in Fig. 1.

\begin{tabular}{|c|c|c|c|c|c|c|}
\hline Interview & First 2 Problems & Middle 2 Problems & Final 2 Problems & \multicolumn{3}{|c|}{ Interviewees } \\
\hline Version & (No Diagramming Prompt) & (Diagram Provided) & (Diagramming Prompt) & Undergrad & Grad & Postdoc/Faculty \\
\hline V1 & Blocks \& Lens & Collision \& E-Potential & Mirrors \& E-Field & Umber & Gale & Felix \\
\hline $\mathrm{V} 2$ & Collision \& E-Potential & Mirrors \& E-Field & Blocks \& Lens & Uri & Gideon & Finn \\
\hline V3 & Mirrors \& E-Field & Blocks \& Lens & Collision \& E-Potential & Uwe & Glenn & Florian, Forest \\
\hline
\end{tabular}

working through the prompts while the interviewer (author MV) observed and answered questions. All interviewees responded to email solicitations at a predominately White R1 university in the US. Student interviewees were financially compensated for their time. We used Zoom's automatic translation feature to generate preliminary transcripts, which we refined during our analysis by referring back to the recorded video files. All participants are referred to by pseudonyms, which all start with the letter $\mathrm{U}$ for the undergraduates, $\mathrm{G}$ for the graduates, and $\mathrm{F}$ for the postdocs and faculty. These different populations were solicited to capture ideas around diagramming from problem solvers across a wide range of experiences.

Data captured in the current study were not intended to be directly comparable with previous data, but rather to help us expand on and nuance our previous findings. Our analysis is focused on the follow-up discussion, not on the diagrams generated by the interviewees during problem solving, though these diagrams were important in grounding the follow-up discussions. The categories of orienting, solving, and communicating, which came from the literature and were observed in our previous study, served as an a priori coding scheme: we first placed quotes from the interviewees into these larger categories, and then used an iterative emergent coding [22] process to identify smaller-grain categories. We did not look for frequencies in the emergent codes, in part due to the semi-structured nature of these conversations: instead, this analysis was intended to capture the breadth of ideas put forward by our interviewees, though we do not claim these categories are exhaustive.

\section{RESULTS}

Diagrams present information in a visual format, which all of our interviewees described as valuable. To understand the many interviewee statements around diagramming, we organize their statements into the broad topics of orienting, solving, and communicating, though many interviewee ideas span multiple categories.

\section{A. Orienting}

Drawing unprompted diagrams to orient to a situation occurred during two-thirds of $\mathrm{MC}$ problems in our previous study. In the current study, interviewees described how diagramming helped them get oriented.

Understanding the Physical Situation: Drawing a diagram after, or even while, reading the problem state- ment helped interviewees get a handle on the physical situation described in the prompt:

Gideon: The first thing I'm thinking about is what is actually happening...like even before I fully read a problem, I start sketching it out.

Finn: I start drawing a diagram even like before I sometimes ask what the problem is asking...to get my mind wrapped around 'what is it saying?'

Understanding the Question: Diagramming also helped interviewees understand what a question is asking:

Umber: I think about [diagramming] as a useful tool and helpful means of trying to get an intuition to what the problem...is asking.

Glenn: I need to draw a diagram... and then the diagram can help me to understand the question, and then make me solve the question.

\section{B. Solving}

As Glenn's quote above suggests, diagrams generated for orienting can then aid in solving the problem. Our interviewees discussed multiple ways in which diagrams support problem-solving.

Storing Information: Diagrams can store information about the problem. Interviewees discussed storing information both given in problem prompts and acquired as they worked though the problem:

Felix: First and foremost, it lets me like store information somewhere where it doesn't have to be in my active brain memory, so I can like use brain power to think about other things, and the other details that I figured out are waiting somewhere for me to retrieve them when I need them.

Other interviewees echoed this idea that the diagram can help reduce cognitive load (an idea consistent with the distributed cognition framework [2, 23]):

Uri: Pictures act as a way to relieve like some of that mental stress.

Organizing Information: Many interviewees discussed how diagrams can organize information:

Florian: A diagram [is] an essential means of organizing sometimes complex spatial information or...parameter space information.

Having information organized in this way can help prevent mistakes later in the problem-solving process: 
Glenn: So I just mark [the friction force between] these two objects, and then that will helped me to, you know, to make everything clear, so [I] won't confuse or mix the numbers.

Prioritizing Information: Many interviewees discussed being intentional about the information they store or organize within their diagrams:

Uwe: I tend to simplify the diagrams as much as I can.

Uri: Pictures should be relatively concise... I don't really feel the need to include numbers in my pictures.

Florian discussed how a diagram might not initially be a place to prioritize information, but that prioritizing the information on a diagram might be worth the work of creating subsequent diagram(s):

Florian: [I] streamline it down to kind of the most essential information... I always draw just the first diagram that comes to mind, and then I kind of refine that... with the essential information.

For other problem-solvers, however, storing and organizing information is enough, and no effort is made to prioritize information:

Finn: I kind of try to put all the pieces of information into the diagram, just so that I know that I understand it.

Supporting and Verifying Solutions: In addition to managing information, interviewees discussed how diagrams can can support algebraic solutions throughout problem solving. Felix described an instance where the diagram helped a solution-approach take shape:

Felix: I drew just because I sort of didn't know what my grand strategy was, so it was it was a natural first step...then [when] what seemed like the calculation of the strategy emerged, the diagram stopped feeling as useful.

Gideon described several contexts where a diagram has supported or confirmed results from calculations:

Gideon: For optics...I've just relied on like the tracing to at least remind me...is it magnified or does it shrink, you know? Does it invert, does it not? [With special relativity], I remember sometimes getting twisted, like backwards, on whether things are contracting or lengthening... and I remember kind of using vectors or pictures to help remind myself of what's supposed to happen.

Uwe and Uri explicitly describe using a diagram to check their final results:

Uwe: At the end [of the Lens problem], like I did [diagram] to double check that the answer made sense in terms of how the image would turn out.

Uri: [With a diagram] it's also, you know, easier to check my work.
Finally, Forest describes when a diagram helped them catch a mistake:

Forest: The diagram makes it very clear that the $z$-component is negative...the $x$-component is positive...whereas the first thing that I wrote wasn't clear on that.... The diagram helped here, but the diagram is sufficiently complicated that it wasn't the first thing that I turned to.

This sentiment about the limitations of some diagrams was shared by other interviewees and is discussed later.

\section{Communicating}

Diagrams can communicate information to others. Many interviewees commented on differences between diagrams meant for themselves and diagrams meant for others, as well as the impact of (for two of the problems) being presented with a diagram.

Communicating to Others: Two of the MC problems and the three diagramming tasks prompted the interviewees to construct clear diagrams. Glenn discusses the impact this had on their diagrams:

Glenn: When you ask me to do the diagram, then I will try to draw the diagram much more clear, you know? In the previous questions, I just need to understand myself, I don't need other people to understand my diagram.

Uwe expressed a similar idea, relevant to teaching:

Uwe: If I had to teach it or show it to someone else, I would definitely make a point to make [the diagram] larger.

Finn described how their Collision diagram contained more information than they might include in a diagram intended for someone else to use:

Finn: This is just not the cleanest diagram, it's kind of a combo of diagram plus shorthand for myself, I guess.

Finally, Forest notes that they value carefully-drawn diagrams as a way of evaluating student understanding:

Forest: "Draw a careful diagram," is much more in line with what I would be looking for if I wanted the students to draw a diagram and demonstrate to my satisfaction that they understand something about ray optics and mirrors.

Given our previous finding that unprompted and prompted diagrams often contain different features $[18$, 19], these quotes help us to better understand some of the reasoning that goes into generating different diagrams for different purposes.

\section{Other: To Draw or not to Draw?}

In addition to discussing the benefits of diagrams, our interviewees also shared reasons for not diagramming.

Physical Context: Many interviewees discussed how for some physical contexts, such as the E-Potential problem, diagrams are unnecessary and not worth the effort: 
Gale: Potential is like a scalar quantity, So I think I didn't really need the drawing.

Gideon: Nothing about which of the variables I'm solving for needs to be clarified by the picture.

Florian: In certain cases, it can be easier just to write down the equations and just solve the math.

While these interviewees expressed that sometimes diagramming is not worth the time and effort, others discussed how, even in instances when a diagram might be helpful, the difficulty of generating the diagram may prevent problem solvers from creating or finishing a diagram:

Forest: It's not easy to visualize [the E-field problem] because it's a three dimensional problem, so the diagram that I'm drawing here, right, doesn't lend itself particularly well to helping keep track.... There's really nothing one can do except to sit down and grunt it out in detail.

Umber discussed how, even when a diagram might be helpful, they would prefer to generate it using a computer:

Umber: Had this [E-field problem] been a homework problem, for instance, I guess I would have considered plotting it in Mathematica or GeoGebra or something like that, because then the vector field would be...easier to actually visually depict.

Problem solvers weigh costs and benefits before diagramming, which likely explains the different frequencies of diagramming across problems in our previous study.

Personal Preferences and Experience: Several interviewees referred to diagramming as being habitual:

Uri: The picture is like a mental shortcut...[but] I've gotten so used to not taking that shortcut that I...have to draw the picture. So it's like, yeah, just habitual.

Gideon: This definitely could have been done without a picture.... I probably wouldn't have drawn it except maybe out of habit.

Others discussed a 'do as I say, not as I do' attitude towards instruction around diagramming:

Felix: I thought about properly drawing, you know, a free body diagram off to the side...which I usually encourage my students to do to keep the diagrams clean and to avoid getting confused.

A similar sentiment was expressed by Uwe (Sec. III C) about how the diagrams they would present when teaching would be "larger" and more clear.

Finally, multiple interviewees discussed how diagramming can be challenging:

Forest: Those tools of perspective drawing aren't available to everybody, that's not what their strength is.

Umber: I will warn that diagrams are not my strong suit.
TABLE II. Interviewees' expressed reasons for diagramming.

\begin{tabular}{ll}
\hline \hline Orient & to situation, to question/task \\
Solve & store info, organize info, prioritize info, sup- \\
& port solutions, verify solutions \\
Communicate & understanding to others \\
Other Factors & personal preference, personal experience. \\
\hline \hline
\end{tabular}

\section{SYNTHESIS AND CONCLUSION}

In this study, we asked physics problem solvers when and why they generate diagrams while problem solving (summarized in Table II). These findings can serve as a basis for further study of student-generated diagrams and inform research and instruction regarding diagrams and other (or multiple) representations: in particular, they can help researchers ask or articulate which of these (or potentially other) uses students in other settings might be seeking or leveraging.

Our findings support our previous claim that instructors should be intentional in how they teach and evaluate diagramming. With myriad reasons for drawing diagrams, our interviewees discussed how different diagram features serve different roles, how diagramming is a skill that requires direction and support to develop, and how helpful problem-solving diagrams are often different from those of communication diagrams (which ties into previous conceptualizations of representation as process versus representation as product [1]). We also again saw little evidence that interviewees considered solving problems geometrically, so instruction around geometric solutions (if valued) needs to be explicit.

As for differences in the populations we interviewed (undergrad, grad, postdoc, and faculty), we found no patterns in why they generated diagrams, though this could be a result of our low $\mathrm{N}$ or the semi-structured nature of the interview conversations, though it is also worth noting that all students were physics majors who had completed at least one full year of physics. One difference that may be of note to researchers: while student and postdoc interviewees jumped straight into solving the problems, our two faculty interviewees, completely unprompted, spent a significant amount of time discussing the pedagogical value of these questions: depending on the research goals, explicit and customized prompting for different populations may be necessary to ensure comparable data sets.

Future work could explore the impact of different diagramming prompts, exploring differences in diagramming among groups of various experience, and exploring the role and effectiveness of diagramming and visualizations for students with visual and other impairments.

\section{ACKNOWLEDGEMENTS}

We thank members of the PER@C group and our interview participants. This work was supported by the CU Department of Physics. 
[1] M. Scaife and Y. Rogers, External cognition: how do graphical representations work?, International Journal of Human-Computer Studies 45, 185 (1996).

[2] T. M. Foster, Implications of Distributed Cognition for PER, in AIP Conference Proceedings (2002).

[3] D. Rosengrant, A. Van Heuvelen, and E. Etkina, Case Study: Students' Use of Multiple Representations in Problem Solving, in AIP Conference Proceedings, Vol. 818 (AIP, Salt Lake City, Utah (USA), 2006) pp. 49-52.

[4] P. B. Kohl, N. D. Finkelstein, L. Hsu, C. Henderson, and L. McCullough, Expert and Novice Use of Multiple Representations During Physics Problem Solving, in AIP Conference Proceedings (AIP, Greensboro (NC), 2007) pp. 132-135.

[5] D. Kirsh, Thinking with external representations, AI \& SOCIETY 25, 441 (2010).

[6] A. F. Heckler, Some Consequences of Prompting Novice Physics Students to Construct Force Diagrams, International Journal of Science Education 32, 1829 (2010).

[7] R. J. Dufresne, W. J. Gerace, and W. J. Leonard, Solving physics problems with multiple representations, The Physics Teacher 35, 270 (1997).

[8] H. H. Tairab and A. K. K. Al-Naqbi, How do secondary school science students interpret and construct scientific graphs?, Journal of Biological Education 38, 127 (2004).

[9] D. E. Meltzer, Student Learning In Upper-Level Thermal Physics: Comparisons And Contrasts With Students In Introductory Courses, in AIP Conference Proceedings, Vol. 790 (AIP, Sacramento, California (USA), 2005) pp. $31-34$.

[10] D.-H. Nguyen, N. S. Rebello, M. Sabella, C. Henderson, and C. Singh, Students' Difficulties in Transfer of Problem Solving Across Representations, in Physics Education Research Conference 2009 (2009) pp. 221-224.

[11] A. Savinainen, A. Mäkynen, P. Nieminen, and J. Viiri, Does using a visual-representation tool foster students' ability to identify forces and construct free-body diagrams?, Physical Review Special Topics - Physics Education Research 9, 10.1103/PhysRevSTPER.9.010104 (2013).

[12] B. Hand and A. Choi, Examining the Impact of Student Use of Multiple Modal Representations in Constructing
Arguments in Organic Chemistry Laboratory Classes, Research in Science Education 40, 29 (2010).

[13] P. B. Kohl and N. D. Finkelstein, Student representational competence and self-assessment when solving physics problems, Physical Review Special Topics Physics Education Research 1, 010104 (2005).

[14] D.-H. Nguyen, E. Gire, N. S. Rebello, C. Singh, M. Sabella, and S. Rebello, Facilitating Students' Problem Solving across Multiple Representations in Introductory Mechanics, in Physics Education Research Conference 2010 (2010) pp. 45-48.

[15] E. Gire and E. Price, Structural features of algebraic quantum notations, Physical Review Special Topics Physics Education Research 11, 10.1103/PhysRevSTPER.11.020109 (2015).

[16] D. McPadden and E. Brewe, Network Analysis of Students' Representation Use in Problem Solving, in Physics Education Research Conference 2015 (2015) pp. 219-222.

[17] R. R. Bajracharya, P. J. Emigh, and C. A. Manogue, Students' strategies for solving a multirepresentational partial derivative problem in thermodynamics, Physical Review Physics Education Research 15, 020124 (2019), publisher: American Physical Society.

[18] M. Vignal and B. R. Wilcox, Comparing Unprompted and Prompted Student-Generated Diagrams, in Physics Education Research Conference 2020 (2020) pp. 551-556.

[19] M. Vignal and B. R. Wilcox, Investigating Unprompted and Prompted Diagrams Generated by Physics MajorsDuring Problem Solving, arXiv:2106.07765 [physics] (2021), arXiv: 2106.07765.

[20] K. A. Ericsson and H. A. Simon, Protocol analysis: Verbal reports as data. (the MIT Press, 1984).

[21] E. Drever, Using Semi-Structured Interviews in SmallScale Research. A Teacher's Guide. (ERIC, 1995).

[22] S. E. Stemler, Content analysis, Emerging Trends in the Social and Behavioral Sciences: An Interdisciplinary, Searchable, and Linkable Resource , 1 (2015).

[23] R. Pea, Distributed Intelligence and design for education, Course Pack for Instructional Applications of Computers (1993). 